\title{
Infección del sitio quirúrgico en pacientes apendicectomizados por técnica abierta y laparoscópica, Cuenca, Ecuador
}

\section{Surgical site infection in patients appendectomed by open and laparoscopic technique, Cuenca, Ecuador}

\author{
Juan Andrés Ávila Narváez*1 ${ }^{1}$, Jorge Eduardo Ávila Narváez ${ }^{2}$, Jessica Gabriel Vásquez Cevallos ${ }^{3}$, Carlos Ivan \\ Aguilar Gaibor ${ }^{4}$, Edison Gustavo Moyano Brito ${ }^{2}$ \\ ${ }^{1}$ Hospital General de Quevedo, Quevedo, Los Ríos, Ecuador \\ ${ }^{2}$ Universidad Católica de Cuenca, Ecuador \\ ${ }^{3}$ Hospital Homero Castanier, Azogues, Ecuador \\ ${ }^{4}$ Hospital José Carrasco Arteaga, Cuenca, Ecuador \\ *jandres2005@gmail.com
}

DOI: https://doi.org/10.26871/killkanasalud.v4i4.748

\begin{abstract}
Resumen
Antecedentes: la infección del sitio quirúrgico puede involucrar tejidos debajo de la piel, órganos o material implantado. Objetivo: establecer la prevalencia y los factores asociados a la infección del sitio quirúrgico en pacientes apendicectomizados, en los hospitales Vicente Corral Moscoso y José Carrasco Arteaga, durante el periodo 2018. Método y materiales: se trata de un estudio analítico transversal. Los datos se extrajeron de la historia clínica, la nota operativa y del reporte de anestesia de 384 pacientes operados por apendicitis aguda. Para determinar los factores asociados se utilizó el estadístico OR (IC95\%), además la significancia estadística se estableció utilizando el modelo de regresión logística binaria con un valor $\mathrm{p}<0,05$. Resultados: la prevalencia de la infección sitio quirúrgico fue del $12 \%$. Se encontraron como factores de riesgo con significancia estadística el antecedente de diabetes (OR 3,2; IC95 \% 1,62 - 6,27; p 0,00), y el tiempo quirúrgico mayor a 1 hora (OR 2,5; IC95 \% 1,0 - 5,9; p 0,04). En cambio, hubo asociación, mas no significancia estadística entre la edad mayor a 29 años (OR 1,5; IC95 \% 0,63 - 3,55; p 0,35), los antecedentes de diabetes (OR 1,7; IC95 \% 0,5 - 5,6; p 0,37), la técnica quirúrgica convencional (OR 1,3; IC95\% 0,6 - 3,01; p 0,37). La obesidad (OR 0,8; IC95 \%; valor p 0,73) no fue un factor asociado. Conclusiones: la prevalencia de infección del sitio quirúrgico en los pacientes apendicectomizados fue mayor a lo reportado que en la literatura, y está asociado a la diabetes y al tiempo quirúrgico mayor a 1 hora.
\end{abstract}

Palabras clave: infección de la herida quirúrgica, infección focal, apendicitis, apendicectomía, laparoscopía.

\begin{abstract}
Background: surgical site infection may involve tissues under the skin, organs, or implanted material. Objective: to establish the prevalence and factors associated with infection of the surgical site in appendectomized patients, in the Vicente Corral Moscoso and José Carrasco Arteaga hospitals, during the 2018 period. Method and materials: this is a cross-sectional analytical study. Data were extracted from the clinical history, the operative note and the anesthesia report of 384 patients operated on for acute appendicitis. To determine the associated factors, the OR statistic (95\% CI) was used, in addition, statistical significance was established using the binary logistic regression model with a p value $<0.05$. Results: the prevalence of surgical site infection was $12 \%$. The risk factors with statistical significance were the history of diabetes (OR 3.2; 95\% CI 1.62 - 6.27; p 0.00), and the surgical time greater than 1 hour (OR 2.5; 95 CI \% 1.0 - 5.9; $p$ 0.04). On the other hand, there was an association, but no statistical significance between the age over 29 years (OR 1.5; $95 \%$ CI 0.63 - 3.55; $p$ 0.35), the history of diabetes (OR 1.7; 95 CI \% 0.5 - 5.6; $p$ 0.37), the conventional surgical technique (OR 1.3; 95\% CI 0.6 - 3.01; p 0.37). Obesity (OR 0.8; 95\% CI; p-value 0.73) was not an associated factor. Conclusions: the prevalence of surgical site infection in appendectomized patients was higher than that reported in the literature, and is associated with diabetes and a surgical time greater than 1 hour.
\end{abstract}

Keywords: surgical wound infection, focal infection, appendicitis, appendectomy, laparoscopy. 


\section{Introducción}

La apendicectomía es uno de los procedimientos quirúrgicos de emergencia más frecuentes en el mundo, y la complicación postoperatoria más común es la infección superficial del sitio quirúrgico (ISSQ), que ocurre especialmente en apendicitis complicada (es decir, gangrenosa y apendicitis perforada. La ISSQ constituye un problema común que afecta los grupos de edad más jóvenes, sólo el 5-10\% de los casos ocurren en la población anciana, la incidencia máxima ocurre entre los 20 a 30 años y hay un ligero predominio de hombres sobre mujeres. ${ }^{1}$

Las infecciones en el sitio quirúrgico son una causa frecuente de morbilidad después de los procedimientos quirúrgicos. Los cocos grampositivos, particularmente los estafilococos causan muchas de estas infecciones, aunque también participan frecuentemente organismos gramnegativos. El riesgo de desarrollar una infección en el sitio quirúrgico está asociado con una serie de factores, que incluyen aspectos del procedimiento quirúrgico en si, como la clasificación de la herida y las variables relacionadas con el paciente, como las afecciones medicas preexistentes. Tanto las medidas no farmacológicas como la profilaxis antimicrobiana para procedimientos seleccionados se utilizan para prevenir el desarrollo de estas infecciones. ${ }^{2}$

En un estudio realizado en Latacunga - Ecuador en el 2015, se encontró que la infección de herida quirúrgica fue la complicación más frecuente $(73 \%)$. La edad de aparición más común fue de 11 a 14 años, hubo predomino del sexo masculino $(57 \%)$ frente al femenino (43\%). El $32 \%$ de los pacientes utilizó analgésicos previos al diagnóstico de Apendicitis Aguda. El factor económico, nivel cultural y lugar de residencia, influyen en la presencia de complicaciones postoperatorias. ${ }^{3}$

En un estudio realizado en Cuenca en el 2009, se recopiló información de 155 pacientes a quienes se realizó apendicetomía convencional. La prevalencia de complicaciones postquirúrgicas fue de $21,9 \%$. La apendicitis se presentó predominantemente en mujeres $(52,3 \%)$, entre los 16 a 30 años $(45,8 \%)$, en residentes de zona urbana $(77,4 \%)$ y en personas con IMC normal $(62,8 \%)$. Los factores asociados con significancia estadística fueron: edad menor a 3 y mayor a 64 años, incisión media infraumbilical, tiempo quirúrgico mayor a 60 minutos, tiempo de evolución mayor a $48 \mathrm{~h}$ y la presencia de fases gangrenosa o perforada en el curso de la apendicitis aguda. La herida quirúrgica fue el sitio de complicación en el 94,1\% de los casos (n: 32). Las complicaciones intraabdominales dieron cuenta del 5,9\%. ${ }^{4}$

Actualmente sabemos los costos que crean las largas estancias de los pacientes complicados en los servicios de hospitalización y los inconvenientes financieros que a la vez estos generan, además, se asocia a esto el uso irracional de la terapia antimicrobiana, que causa la resistencia de bacterias de hábitat nosocomial y que van a repercutir en la hospitalización prolongada en el paciente. ${ }^{5}$

Foster D, ${ }^{6}$ encontró una tasa de ISQ del $17,9 \%$ infecciones por apendicectomías abiertas, y 8,8\% infecciones por apendicectomías. Las tasas de ISQ fueron más altas en la apendicitis complicada y cuando no se especificó el uso preoperatorio de antibióticos.

Aranda $\mathrm{J}^{7}$ encontró, que la ISQ global fue $13,4 \%$ en AA, y en AL $14 \%$. Hubo asociación estadística entre el abordaje laparoscópico/ acceso abierto e ISQ, especialmente en pacientes de alto riesgo $\mathrm{p}<0,000$.

Ingraham $A,{ }^{8}$ en el año 2010, demostró en un total de 32.683 pacientes, $(76,4 \%)$ se sometieron a AL, y $(23,6 \%)$ a AA, que los pacientes que se sometieron a AA eran significativamente mayores con más comorbilidades en comparación con los que se sometieron a AL. Todos los pacientes tratados con AL tenían significativamente menos probabilidades de desarrollar complicaciones infecciosas individuales, excepto la ISQ del espacio orgánico. Entre los pacientes con apendicitis complicada la ISQ del espacio orgánico fue significativamente más común después de la AL (6,3\% frente al 4,8\%, OR 1,35; IC95 \% 1,05 - 1,73).

Cho, en el año 2014, indicó que los pacientes mayores de 55 años presentaron infección del sitio quirúrgico en el $17,4 \% .{ }^{9}$ La ISQ, puede duplicar el tiempo que un paciente permanece en el hospital y, por lo tanto, aumentar los costos de la atención médica. Los principales costos adicionales están relacionados con la reintervención quirúrgica, requerimientos médicos y de enfermería adicionales, así como el uso extra de medicamentos. ${ }^{10}$

Una revisión sistemática desde 1998 hasta abril de 2014, halló beneficio en el baño (todo el cuerpo) con jabón (antimicrobiano o no antimicrobiano) o con un agente antiséptico al menos la noche previa a la cirugía. La profilaxis antimicrobiana se debe administrar solo cuando se indique en función de las guías de práctica clínica publicadas, con el tiempo adecuado para lograr una concentración bactericida en el suero y los tejidos en el momento de la incisión. La preparación de la piel en el quirófano debe realizarse con un agente a base de alcohol, a menos que esté contraindicado. Para procedimientos limpios y limpios contaminados, no deben administrarse dosis adicionales de agentes antimicrobianos profilácticos, incluso en presencia de un drenaje. No se deben aplicar agentes antimicrobianos tópicos a la incisión quirúrgica. Durante la cirugía, debe llevarse a cabo control glucémico, con objetivo de niveles de glucosa en la sangre menores a $200 \mathrm{mg} / \mathrm{dL}$, y debe procurarse normotermia en todos los pacientes. ${ }^{11}$

La complicación más frecuente posterior a una apendicectomía es la ISQ, que se ha visto en 3,6\% para cirugía laparoscópica y 7,3\% para cirugía abierta; el absceso intraabdominal se presenta en $1,6 \%$ y $0,6 \%$ para cirugía laparoscópica y abierta respectivamente. ${ }^{12}$

Suh y colaboradores, realizaron en Corea un estudio que sirvió para la comparación entre la apendicectomía laparoscópica y la abierta en términos de ISQ, se hallaron registros médicos de 749 pacientes (420 hombres; edad media, 33 años) a los que se realizó apendicectomía (431 abordajes abiertos vs 318 laparoscopías) entre el 1 de septiembre de 2008 y el 29 de abril de 2010. Los resultados de este es- 
tudio sugieren que la Apendicetomía Laparoscópica puede conducir a una estancia hospitalaria más corta y un riesgo menor de ISQ incisional superficial que la Apendicectomía Abierta. ${ }^{13}$ Sin embargo, un tiempo de espera para la cirugía mayor de 6 horas se asoció con un aumento de ISQ como un factor independiente. ${ }^{14}$

Los factores que predisponen a la ISQ son varios, y para evitarlos, la American Society of Anesthesiologist (ASA) y la National Nosocomial Infección Surveillance Systema (NISS), clasifica a los individuos de acuerdo a su cuadro clínico, a la potencial presencia de microorganismos, a la posible contaminación de la herida quirúrgica, tiempo de duración de la cirugía y tipo de cirugía; además, considera la existencia de otros factores como: el estado nutricional, el consumo de tabaco, antecedentes de transfusiones sanguíneas, el aseo previo al procedimiento quirúrgico, y enfermedades crónicas preexistentes. Esto se consideró, con la evidencia en la literatura sobre la relación entre estos factores y la ISQ.

En Etiopía, en donde se evaluó del uso de profilaxis antimicrobiana y tasa de ISQ. Recolectaron muestras de heridas y las procesamos en base a un procedimiento operativo estándar, y se realizó una prueba de sensibilidad a los antibióticos con difusión por disco. Se concluyó que la tasa de ISQ fue relativamente alta. Se encontró que Klebsiella pneumoniae es el agente más causante de la ISQ. Los organismos que causan ISQ fueron sensibles a los agentes antimicrobianos de uso común en dicho hospital. ${ }^{9}$

Por los cambios asociados al envejecimiento, las personas de la tercera edad tienen una probabilidad de 3 a 5 veces más de tener una infección hospitalaria en comparación con la población de menor edad; por ello, este grupo de edad tiene más posibilidades de desarrollar una ISQ. ${ }^{15}$

Gissen, ${ }^{16}$ El sexo masculino representó el $54,3 \%$ del grupo de estudio. La mediana de edad fue de 31 (18-46) años. La apendicectomía laparoscópica se realizó en el $78,9 \%(\mathrm{n}=502)$ de los pacientes. Diez operaciones que comenzaron por vía laparoscópica se convirtieron en un procedimiento abierto (2\%). En 8 casos, el procedimiento laparoscópico no pudo continuar debido a una anatomía poco clara. En 2 casos, un infiltrado apendicular hizo imposible continuar de manera segura con la laparoscopia. Treinta y cinco por ciento $(n=225)$ de los pacientes tenían apendicitis compleja, de los cuales 110 pacientes tenían un apéndice perforado. En esta investigación se encontró que la edad igual o mayor a 31 años (p 0,87), el sexo masculino ( $\mathrm{p} 0,95)$ no tuvieron relación estadística con la ISQ.

Kasatpibal N, y cols., ${ }^{17}$ demostró que la edad igual o mayor a 40 años fue un factor asociado, pero sin significancia estadística (RR 2,23; IC95 \% 0,85 - 5,83) para desarrollar ISQ. A diferencia de Brill y cols., ${ }^{18}$ quienes realizaron un análisis retrospectivo en 11,662 ingresos en 22 hospitales en el año 2008. El análisis bivariante demostró que el sexo masculino (OR 1,$9 ; 1,19-1,90)$ y la edad $\geq 75$ años (OR 2,96; IC95 \% 2,25-3,90) fueron factores de riesgo para la ISQ.
Billoro B, y cols., ${ }^{19}$ en el año 2019, realizó un estudio donde se extrajeron los datos de la historia clínica, la nota operativa y de anestesia por observación directa aplicando un cuestionario semiestructurado. Se reveló como factor asociado a la ISQ la edad mayor a 40 años (OR 7,11; IC95 \% 1.47-15.022).

La obesidad como factor de riesgo para la ISQ, es debido a la necesidad de incisiones más amplias; además, el tejido subcutáneo adiposo abundante tiene la peor vascularización, y por ello, la acción de antibióticos profilácticos no es adecuada. ${ }^{15}$

La obesidad es un factor de riesgo de ISQ después de procedimientos abdominales; sin embargo, faltan datos que caractericen el riesgo de ISQ en pacientes obesos durante los procedimientos abdominales. Winfield y cols., ${ }^{20}$ en el 2016 mediante un analisis de regresión logística confirmó que la obesidad y la obesidad mórbida se asocian de forma independiente con el desarrollo general de ISQ, específicamente en un procedimiento quirúrgico limpio (Obesidad OR 1,7; obesidad mórbida OR 2,5) siendo esta relación estadísticamente significativa (p 0,001); y con un procedimiento limpio contaminado (obesidad OR 1,2; obesidad mórbida OR 1,3, p 0,001). En conclusión: la obesidad se asocia con un mayor riesgo de ISQ en general, específicamente en procedimientos abdominales limpios y contaminados, y esta relación es independiente de la diabetes mellitus.

Noorit ${ }^{21}$ encontró una relación significativa entre el IMC y la ISQ (p 0,023), así también, con el grosor de la grasa subcutánea (p 0,033).

La hiperglucemia favorece las infecciones producidas por bacterias y hongos. Las alteraciones vasculares propias de la enfermedad disminuyen los mecanismos de defensa del organismo. Por ello, los niveles de glucosa superiores a 200 o $230 \mathrm{mg} / \mathrm{dl}$ durante la intervención o en el postoperatorio inmediato, aumenta el riesgo de tener una ISQ. En resumen, las personas con diabetes son más propensas a las ISQ, de ahí que los protocolos indican una infusión continua con insulina. ${ }^{15}$ Noorit $^{21}$ entre 607 pacientes, determinó con el análisis bivariado que la Diabetes fue un factor de riesgo para la ISQ en pacientes apendicectomizados (OR 2,6; IC $95 \%$ 1,2, 5,9; p 0,005).

La piel se cierra en apendicectomía abierta tradicionalmente con pocas suturas no absorbibles interrumpidas. El uso de este antiguo método se basa en la sugerencia de que esta técnica disminuye las infecciones de la herida. ${ }^{22}$ Brill y cols., ${ }^{18}$ en cambio, reportaron que la técnica laparoscópica (OR 0,32; IC95 \% 0,21-0,52) resultó ser un factor protector para ISQ.

Xiao Y,. ${ }^{23} \mathrm{La}$ incidencia de ISQ general fue de $6,2 \%$. En comparación con la AA, la AL se asoció con menores incidencias de ISQ general e incisional (4,5 vs $6,7 \%$, p $<0,001$; y 1,9 vs 4,2\%, p <0,001). El análisis de regresión logística multivariante, se encontró que AL se asociaba con ISQ global [OR 1,24 (IC95\% 1,03-1,70); $\mathrm{p}=0.04$ ] o ISQ incisional [OR 1,32 (IC $95 \%$ 1,10-1.,68); p = 0,01]. 
Jaschinski $\mathrm{T}^{24}$ encontró que en los adultos, las ISQ fueron menos probables después de AL (OR 0,42, IC $95 \%$ $0,35-0,51 ; 63)$, pero la incidencia de abscesos intraabdominales aumentó después de AL (OR 1,65, $95 \%$ IC 1,12 - 2,43). La duración de la estancia hospitalaria se acortó un día después de AL (DM -0,96; IC95\%: -1,23 a $0,70 ; 46)$. En los niños, abscesos intraabdominales después de AL (OR 0,54, IC95 \% 0,24 - 1,22). Sin embargo, las infecciones de la herida fueron menos probables después de AL (OR 0,25, IC $95 \%$ 0,15 - 0,42) y la duración de la estancia hospitalaria se acortó en 0,8 días después de AL (MD -0,81, IC 95\%: -1,01 a -0,62).

Gissen, ${ }^{16}$ no demostró, en los 637 pacientes incluidos en su estudio que el tiempo quirúrgico se relacione con la ISQ (p 0,30). En cambio, Kasatpibal N, y cols., ${ }^{17}$ en su estudio de cohorte prospectivo en ocho hospitales de Tailandia, entre 2139 pacientes con apendicectomía, identificaron 26 casos de ISQ, lo que arroja una tasa de 1,2 infecciones / 100 operaciones. El tiempo quirúrgico mayor a 1 hora fue un factor de riesgo para la ISQ (RR 2,75; IC95 \% 1,24 - 6,11). Resultados similares estableció Noorit ${ }^{21}$ al determinar que el tiempo operatorio $>75$ min (OR 3,4; IC95 \% 1,8, 6,5; p $0,001)$ fue un factor de riesgo para ISQ.

Billoro B, y cols., ${ }^{19}$ también determinó que el tiempo de operación más de $1 \mathrm{~h}$ fue un factor de riesgo asociado a la ISQ (OR 2,57; IC95 \% 1,56-4,23; p 0,012).

\section{Metodología}

Tipo de estudio: se realizó un estudio de tipo analítico transversal.

Universo: historias clínicas de pacientes de ambos sexos, de todas las edades, con antecedentes de apendicitis aguda, que fueron operados por la técnica convencional o laparoscópica en el Hospital Vicente Corral Moscoso y José Carrasco Arteaga, Cuenca desde el periodo enero 2018 a mayo del 2019.

Muestra: para el cálculo de la muestra se utilizó la fórmula para una población infinita, considerando el $50 \%$ de infecciones del sitio quirúrgico reportado por Noorit P. ${ }^{21}$ con un nivel de confianza del $95 \%$, y el margen de error del $5 \%$. Las 384 historias clínicas se seleccionaron por muestreo aleatorio simple del registro del programa Excel, perteneciente a las instituciones de salud descritos.

Procedimientos para la recolección de información: se solicitó los permisos pertinentes para acceder a la información en los Hospitales Vicente Corral Moscoso y José Carrasco Arteaga. Se identificó las historias clínicas de las personas que cumplieron los criterios de inclusión y exclusión, previa selección por muestreo aleatorio simple.

Se creó un formulario de recolección de los datos, el cual fue validado por el director de la tesis, el asesor metodológico y un experto en el área.

Plan de tabulación y de análisis estadístico: se realizó un análisis descriptivo de cada una de las variables de estudio tanto en escala continua y nominal con medidas de tendencia central y dispersión. La prevalencia se calculó tomando en cuenta el número de casos de infecciones del sitio quirúrgico, dividido por el número de personas que constituyeron la muestra de estudio durante un periodo de tiempo determinado. Para establecer los factores asociados de infección de herida quirúrgica en pacientes apendicectomizados, empleó los estadísticos: Chi-cuadrado, Odds Radio con su Intervalo de Confianza del $95 \%$ (IC95\%), la hipótesis se aceptó con el valor de $\mathrm{p}<0,05$.

Procedimientos y aspectos éticos: los datos del paciente por medio de codificación de los mismos con las iniciales de sus nombres y apellidos. No hubo riesgo para los pacientes ya que fueron revisiones de historias clínicas. Esta investigación no tuvo ningún beneficio directo para los pacientes; sin embargo, se espera que estos resultados sean utilizados para mejorar la atención de los pacientes que sean intervenidos en un futuro por apendicitis aguda. Declaramos que no existió conflictos de interés. Toda la información recolectada se utilizó estrictamente con la finalidad de realizar esta investigación sin ser utilizada para otro fin.

\section{Resultados}

Tabla 1: Características sociodemográficas y estado nutricional de la población estudiada. Hospitales Vicente Corral Moscoso y José Carrasco Arteaga. Cuenca, año 2019

\begin{tabular}{lll}
\hline Variables sociodemográficas & $\mathbf{n ~ 3 8 4}$ & $\%$ \\
\hline Edad Media 40 \pm 16 (DS)mm & & \\
\hline Adolescente & 16 & 4,2 \\
Juventud plena & 87 & 22,7 \\
Adulto joven & 58 & 15,1 \\
Adulto maduro & 192 & 50,0 \\
Adulto mayor & 31 & 8,1 \\
\hline Sexo & & \\
\hline Femenino & 223 & 58,1 \\
Masculino & 161 & 41,9 \\
\hline Ocupación & & \\
\hline Estudian & 92 & 24,0 \\
QQDD & 84 & 21,9 \\
Comercia & 35 & 9,1 \\
Empleado & 15 & 3,9 \\
Chofer & 13 & 3,4 \\
Mecánico & 13 & 3,4 \\
Arquitecto & 10 & 2,6 \\
Panadero & 9 & 2,3 \\
Modista & 8 & 2,1 \\
Secretaria & 6 & 1,6 \\
Empleada & 5 & 1,3 \\
Ingeniero & 5 & 1,3 \\
Médico & 5 & 1,3 \\
Profesor & 5 & 1,3 \\
Constructor & 4 & 1,0 \\
Otros & 75 & 20,4 \\
\hline Estado nutricional & 3 & 0,8 \\
\hline Bajo peso & 120 & 46,6 \\
Peso normal & 179 & 7,0 \\
Sobrepeso & 51,3 \\
Obesidad tipo I & 27 & 1,0 \\
Obesidad tipo II & 4 & \\
Obesidad Grado III & & \\
\hline & & \\
\hline
\end{tabular}


El sexo femenino fue el más reportado (6/10), con una edad promedio de 40 años \pm 16 (DS); siendo el grupo de edad más representativo los adultos maduros (edad $\geq$ 35 hasta 64 años) 5/10; 5 de cada 10 participantes fueron casados, y de ocupación estudiantes, e igualmente 5 de cada 10 pacientes tenían sobrepeso (tabla 1 ).

Tabla 2: Prevalencia de infección de herida quirúrgica en pacientes apendicectomizados, según el procedimiento quirúrgico utilizado. Hospitales Vicente Corral Moscoso y José Carrasco Arteaga. Cuenca, año 2019

\begin{tabular}{lccc}
\hline \multirow{2}{*}{$\begin{array}{c}\text { Tipo } \\
\text { apendicectomía }\end{array}$} & \multicolumn{2}{c}{ Infección de herida quirúrgica } & \multirow{2}{*}{ Total } \\
\cline { 2 - 3 } & $\mathrm{Si}$ & No & \\
\hline Laparoscópica & $30(7,8 \%)$ & $242(63,0)$ & $272(70,8)$ \\
Convencional & $17(4,4 \%)$ & $95(24,7)$ & $112(29,2)$ \\
Total & $47(12,2 \%)$ & $337(87,8 \%)$ & $384(100 \%)$ \\
\hline
\end{tabular}

La prevalencia de infección de herida quirúrgica, en los 384 casos de pacientes apendicectomizados fue del 12,2\% (n 47). De este porcentaje se puede indicar que 1 de cada 10 pacientes operado por vía laparoscópica, podría tener una infección de la herida quirúrgica (tabla 2).
Tabla 3: Prevalencia de infección de herida quirúrgica en pacientes apendicectomizados según la fase apendicular. Hospitales Vicente Corral Moscoso y José Carrasco Arteaga. Cuenca, año 2019

\begin{tabular}{lccc}
\hline \multirow{2}{*}{$\begin{array}{c}\text { Fase } \\
\text { apendicular }\end{array}$} & \multicolumn{2}{c}{ Infección de herida quirúrgica } & \multirow{2}{*}{ Total } \\
\cline { 2 - 3 } & $\mathrm{Si}$ & No & \\
\hline Congestiva & $4(8,50)$ & $25(7,40)$ & $29(7,60)$ \\
Supurativa & $15(31,90)$ & $95(28,20)$ & $110(28,60)$ \\
Necrótica & $19(40,40)$ & $132(39,20)$ & $151(39,30)$ \\
Perforada & $9(19,10)$ & $85(25,20)$ & $94(24,50)$ \\
Total & $47(100)$ & $337(100)$ & $384(100)$ \\
Chi cuadrado de Pearson 0,903087 (valor p 0,824683$)$ & \\
\hline
\end{tabular}

De los 47 casos con infección de herida quirúrgica, independiente del procedimiento, la fase necrótica fue la más frecuente, ya que 4 de cada 10 paciente con una apendicitis en fase necrótica puede desarrollar infección del sitio quirúrgico, sin ser esta relación estadísticamente significativa $(\mathrm{p}>0,05)$ (tabla 3$)$.

Tabla 4: Factores asociados a la infección del sitio quirúrgico, en pacientes apendicectomizados del Hospitales Vicente Corral Moscoso y José Carrasco Arteaga, Cuenca, año 2018

\begin{tabular}{|c|c|c|c|c|c|c|}
\hline \multirow{2}{*}{$\begin{array}{c}\text { Factores } \\
\text { asociados }\end{array}$} & \multicolumn{6}{|c|}{ Infección del sitio quirúrgico } \\
\hline & $\begin{array}{c}\text { Si n } 47 \\
(12,2)\end{array}$ & $\begin{array}{c}\text { No n } 337 \\
(87,8)\end{array}$ & $\begin{array}{l}\text { Total n } 384 \\
\text { (100) }\end{array}$ & OR & IC95\% & Valor $\mathrm{p}$ \\
\hline \multicolumn{7}{|c|}{ Edad mayor 29 años } \\
\hline $\mathrm{Si}$ & $34(8,90)$ & $219(57,00)$ & $253(65,90)$ & \multirow{2}{*}{1,4} & \multirow{2}{*}{$0,71-2,77$} & \multirow{2}{*}{0,32} \\
\hline No & $13(3,40)$ & $118(30,70)$ & $131(34,10)$ & & & \\
\hline \multicolumn{7}{|l|}{ Obesidad } \\
\hline $\mathrm{Si}$ & $13(3,40)$ & $72(18,80)$ & $85(22,10)$ & \multirow{2}{*}{1,4} & \multirow{2}{*}{$0,70-2,80$} & \multirow{2}{*}{0,33} \\
\hline No & $34(8,90)$ & $265(69,00)$ & $299(77,90)$ & & & \\
\hline \multicolumn{7}{|l|}{ Diabetes } \\
\hline $\mathrm{Si}$ & $16(4,20)$ & $47(12,20)$ & $63(16,40)$ & \multirow{2}{*}{3,2} & \multirow{2}{*}{$1,62-6,27$} & \multirow{2}{*}{0,00} \\
\hline No & $31(8,10)$ & $290(75,50)$ & $321(83,60)$ & & & \\
\hline \multicolumn{7}{|c|}{ Técnica quirúrgica convencional } \\
\hline $\mathrm{Si}$ & $17(4,40)$ & $95(24,70)$ & $112(29,20)$ & \multirow{2}{*}{1,4} & \multirow{2}{*}{$0,76-1,94$} & \multirow{2}{*}{0,25} \\
\hline No & $30(7,80)$ & $242(63,00)$ & $272(70,80)$ & & & \\
\hline \multicolumn{7}{|c|}{ Tiempo quirúrgico mayor a 1 hora } \\
\hline $\mathrm{Si}$ & $26(6,80)$ & $132((34,40)$ & $158(41,10)$ & \multirow{2}{*}{1,9} & \multirow{2}{*}{$1,04-1,55$} & \multirow{2}{*}{0,03} \\
\hline No & $21(5,50)$ & $205(53,40)$ & $226(58,90)$ & & & \\
\hline
\end{tabular}

Se observó que hubo asociación, mas no significancia estadística, entre la infección del sitio quirúrgico con la edad mayor a 29 años (OR 1,4; IC95\% 0,71 - 2,77; p 0,32), la obesidad (OR 1,4; IC95\% 0,70 - 2,80; p 0,73), y la técnica quirúrgica convencional (OR 1,4; IC95\% 0,76
- 1,94; p 0,25); en cambio, los antecedentes de diabetes (OR 3,2; IC95 \% 1,62 - 6,27; p 0,00) y el tiempo quirúrgico mayor a 1 hora fueron factores de riesgo para la ISQ (OR: 1,9; IC95 \% 1,04 - 1,55; p 0,03) (Tabla 4). 
Tabla 5: Análisis Multivariantes de los factores asociados a las infecciones del sitio quirúrgico en pacientes apendicectomizados del Hospital Vicente Corral Moscoso y José Carrasco Arteaga, Cuenca 2018

\begin{tabular}{lcccccc}
\hline & B & $\begin{array}{c}\text { Error } \\
\text { estándar }\end{array}$ & Wald & gl & Sig. & Exp(B) \\
\hline$>29$ años &, 174 &, 364 &, 229 & 1 &, 632 & 1,190 \\
Obesidad &, 246 &, 374 &, 434 & 1 &, 510 & 1,279 \\
Diabetes & 1,098 &, 363 & 9,135 & 1 &, 003 & 2,997 \\
Tiempo quirúrgico $>1$ hora &, 702 &, 323 & 4,715 & 1 &, 030 & 2,017 \\
Técnica convencional &, 168 &, 350 &, 231 & 1 &, 631 & 1,183 \\
\hline
\end{tabular}

Las variables que demostraron relación significativa fueron el antecedente de diabetes, y el tiempo quirúrgico mayor a 1 hora; la edad mayor a 29 años, la obesidad, y la técnica convencional no tuvieron significancia estadística. El test de Wald, identifica que el antecedente de diabetes es el factor que más aporta para que ocurra una infección del sitio quirúrgico en pacientes posapendicectomía independientemente de la técnica quirúrgica, pero, esto siempre y cuando estén presentes los otros factores (tabla 5).

\section{Discusión}

De las 384 historias clínicas de los pacientes apendicectomizados que formaron parte de nuestro estudio, el 58,1\% pertenecen al sexo femenino; con una edad promedio de $40 \pm 16$ (DS) años; los grupos más representativos fueron los adultos maduros (edad $\geq 35$ hasta 64 años) en el $50 \%$ de los casos; el 49,7\% estaban casados; de ocupación estudiantes en el 24,0\%; y el 46,6\% con sobrepeso (tabla $1)$, resultados que difieren con la investigación realizada por Giesen ${ }^{16}$ donde reportó que el $54,3 \%$ fueron de sexo masculino con una edad promedio de 31 años; Aranda $^{7}$ en el año 2014 en España en un grupo de 868 pacientes reporta que el sexo masculino representó el $57 \%$ siendo la edad promedio 29,5 años; Cedillo ${ }^{25}$ indica que el sexo masculino representan el 58,1\% siendo el grupo mayoritario comprendido entre los 18-30 años 74,8 \%; Guachún ${ }^{26}$ encontró predominio de sexo masculino del $58,1 \%$ con edad promedio de 30,43 años. El 57,65 \% vive en área urbana, en relación 1,4:1 de los del área rural. En contraposición con Cedillo, ${ }^{25}$ quien indica que el $51 \%$ de pacientes vive en el área rural. Las diferencias pueden deberse a las características propias de cada región.

La prevalencia de infección de herida quirúrgica fue del $12,2 \%$, de estos casos el 7,8 \% fueron por cirugía laparoscópica, el 4,4\% por la convencional (tabla 2). Esto se puede comparar con el trabajo de Foster, ${ }^{6}$ donde la prevalencia fue del 22, 25 y $22 \%$ respectivamente. La prevalencia más alta encontrada fue en Nigeria del $36 \%$. Se encontraron prevalencias más bajas como Aranda ${ }^{7}$ con una prevalencia del $13,47 \%$. Sohn ${ }^{27}$ en el 2015 en Alemania, indica una prevalencia del $13,4 \%$. Cho ${ }^{28}$ en el 2014 en Korea en 101 pacientes, reporta una prevalencia de $12,8 \%$, Cedillo $^{25}$ muestra una prevalencia de $10,5 \%$ en apendicitis aguda no complicada; Noorit ${ }^{21}$ en el 2018 en Tailandia, presenta una prevalencia del $8,7 \%$. Giesen ${ }^{16}$ en el 2016, en un trabajo con 637 pacientes en los Países Bajos, reportó la prevalencia del $6,6 \%$. Xiao ${ }^{23}$ reporta que la prevalencia es del $6,2 \%$. Wu ${ }^{13}$ encontró una prevalencia de $4,9 \%$ de infección del sitio quirúrgico, y la más baja fue de Fukuda ${ }^{29}$ obtuvo una prevalencia del 4,5\%. Las diferencias significativas, pueden corresponder a la cultura y al nivel económico de cada país y al acceso de las técnicas quirúrgicas.

Aranda $^{7}$ en el año 2014, reportó una infección del sitio quirúrgico del $14 \%$ por vía laparoscópica; por vía convencional la infección del sitio quirúrgico fue del $13 \%$. En contraposición Giesen ${ }^{16}$ en el año 2016, en un trabajo con 637 pacientes, reportó que la apendicectomía laparoscópica fue del 78,9\% con conversión del $2 \%$, y prevalencia de infección del sitio quirúrgico fue del 6,18\%. Fukuda ${ }^{29}$ en el 2016, en un estudio de 2074 pacientes, determinó que el $39,29 \%$ de los pacientes fueron operados por vía laparoscópica y el 3,43\% presentó infección del sitio quirúrgico. Igualmente, las diferencias pueden deberse a las regiones donde se realizaron los estudios.

En relación a la fase de la apendicectomía, la necrótica fue la más encontrada en este estudio, siendo $40,4 \%$ en los casos de ISQ, y de estos (tabla 3). Una apendicitis complicada que incluye fase necrótica, perforada con y sin peritonitis localizada o generalizada incrementa 3,2 veces más la probabilidad de presentar infección del sitio quirúrgico OR 3,21 (IC95 \% 1,3-7,91 p 0,008), asociación que también se encontró en los trabajos de $\mathrm{Xiao}^{23}$ en el 2014, donde la apendicitis perforada tiene OR 1.69 (IC95\% 1.44-1.97 p 0.01); Wu, ${ }^{24}$ encontró OR 3.7 (IC95 \% 1.6- 8.3 p 0.001), y Giesen, ${ }^{16}$ OR 4,09 (IC95\% 2.04-8.20), datos que se asemejan con este estudio.

Hubo asociación más no significancia estadística, entre la infección del sitio quirúrgico en pacientes apendicectomizados con la edad mayor a 29 años (OR 1,4; IC95 \% 0,71 - 2,77; p 0,32), la obesidad (OR 1,4; IC95 \% 0,70 - 2,80; p 0,73 ) y la técnica quirúrgica convencional (OR 1,4; IC95\% 0,76 - 1,94; p 0,37); sin embargo, los antecedentes de diabetes (OR 3,2; IC95\% 1,62 - 6,27; p 0,00) y el tiempo quirúrgico mayor a 1 hora, fueron factores de riesgo para la ISQ (OR: 1,9; IC95 \% 1,04 - 1,55; p 0,03). Comparando con los resultados de Aranda, ${ }^{7}$ el autor no encontró diferencia significativa entre la infección del sitio quirúrgico y la apendicectomía laparoscópica o convencional; $\mathrm{Wu}^{13}$ igualmente, no encontró asociación significativa; así mismo, Giesen ${ }^{16}$ no reportó diferencia estadística; y Fukuda ${ }^{29}$ tampoco encontró asociación con la vía de abordaje, pero 
halló que la duración de la cirugía más allá de 1 hora presenta un OR 1.72 (IC95 \% 1,33-2,22). En contraposición $\mathrm{Xiao},{ }^{23}$ quien reporta que la apendicectomía convencional se asocia con la infección del sitio quirúrgico y es estadísticamente significativo con OR 1,24 (IC95 \% 1,03-1,70; p 0.04 ) estos resultados se asemejan con esta investigación.

El análisis multivariado, al realizar una regresión logística bivariada, las variables que demostraron relación significativa fueron el antecedente de diabetes, y el tiempo quirúrgico mayor a 1 hora; la edad mayor a 29 años, la obesidad, y la técnica convencional no tuvieron significancia estadística (tabla 5). Estos datos difieren con los de Xiao $\mathrm{Y}^{23}$ donde usó un modelo de regresión logística multivariante para evaluar los factores de riesgo. El análisis encontró solamente que la apendicectomía laparoscópica se asociaba con la ISQ global [OR 1,24 (IC95 \% 1,03-1,70); p $=0.04]$ o ISQ incisional [OR 1,32 (IC $95 \%$ 1,10-1.,68); p $=0,01]$.

En resumen, en esta investigación se demostró que el antecedente de diabetes y el tiempo quirúrgico mayor a 1 hora fueron condiciones relacionadas a la infección del sitio quirúrgico en pacientes posapendicectomías por vía convencional y laparoscópica. Esto se puede explicar por el hecho de que los pacientes con diabetes alteran el $\mathrm{pH}$ de 7,4 a un valor más alcalino, favoreciendo con ello a que las bacterias consideradas saprofitas se tornen patógena; además, el tiempo quirúrgico mayor a lo considerado, exhibe por más tiempo a los tejidos a u ambiente inadecuado exponiendo las estructuras a una posible contaminación. El aporte que dan estos resultados es que, el conocer estos factores, podrán contribuir en la toma de las medidas necesarias para evitar las complicaciones posquirúrgicas futuras. El inconveniente presentado en la realización de este trabajo, fue encontrar los datos completos en las historias clínicas de los pacientes.

\section{Conclusiones}

La prevalencia de infección del sitio quirúrgico en este estudio es menor a lo comparable con la prevalencia de los países en vías de desarrollo, y es mayor comparado con los países desarrollados. En relación a los factores asociados, no hubo significancia estadística entre la edad de 29 años, obesidad, y la técnica quirúrgica; a diferencia, que los antecedentes de diabetes, y el tiempo quirúrgico mayor a 1 hora si fue un factor de riesgo para la ISQ.

\section{Fuente de Financiamiento}

Este estudio es autofinanciado.

\section{Conflicto de Intereses}

No existen conflictos personales, profesionales, financieroso de otro tipo.

\section{Consentimiento Informado}

Los autores cuentan con el consentimiento informado de los pacientes para la investigación, la publicación del caso 12 y sus imágenes.

\section{Referencias Bibliográficas}

1. De Vries FEE, et al. A systematic review and metaanalysis including GRADE qualification of the risk of surgical site infections after prophylactic negative pressure wound therapy compared with conventional dressings in clean and contaminated surgery: Medicine (Baltimore). 2016 Sep;95(36): e4673. Disponible en: https://www.ncbi. nlm.nih.gov/pubmed/27603360

2. Teixeira PG, et al. Appendectomy timing: waiting until the next morning increases the risk of surgical site infections. Ann Surg. 2012 Sep;256(3):538-43

3. Ayora J. Complicaciones postapendicectomía convencional y factores asociados, Hospital Vicente Corral Moscoso, período agosto 2008-enero 2009, Cuenca 2009. Repositorio Institucional Universidad de Cuenca. Disponible en: http://dspace.ucuenca.edu.ec/handle/123456789/ 18981

4. Van Rossem CC. et al. Duration of antibiotic treatment after appendicectomy for acute complicated appendicitis. Br J Surg. 2014 May;101(6):715-9. doi: 10.1002/bjs.9481. Epub 2014 Mar 26

5. Davis C, Trevatt A, Dixit A, Datta V. Systematic review of clinical outcomes after prophylactic surgery. Ann R Coll Surg Engl. 2016 Jul;98(6):353-7.Disponible en: https:// www.ncbi.nlm.nih.gov/pmc/articles/PMC5209958/

6. Foster D, Kethman W, Cai LZ, Weiser TG, Forrester JD. Surgical Site Infections after Appendectomy Performed in Low and Middle Human Development-Index Countries: A Systematic Review. Surg Infect (Larchmt). 2018 Apr;19(3):237-44.

7. Aranda-Narváez JM, Prieto-Puga Arjona T, GarcíaAlbiach B, Montiel-Casado MC, González-Sánchez AJ, Sánchez-Pérez B, et al. Infección de sitio quirúrgico tras apendicectomía urgente: tasa global y tipo según la vía de abordaje (abierta/laparoscópica). Enferm Infecc Microbiol Clin. 2014 Feb 1;32(2):76-81.

8. Ingraham AM, Cohen ME, Bilimoria KY, Pritts TA, Ko CY, Esposito TJ. Comparison of outcomes after laparoscopic versus open appendectomy for acute appendicitis at 222 ACS NSQIP hospitals. Surgery. 2010 Oct 1;148(4):625-35; discussion 635-7.

9. Instituto Nacional de Estadísticas y Censos. Anuario de Estadísticas de Salud: Camas y Egresos Hospitalarios 2018. Disponible en: https://www.ecuadorencifras.gob.ec/ camas-y-egresos-hospitalarios/

10. National Collaborating Centre for Women's and Children's Health (UK). Surgical Site Infection: Prevention and Treatment of Surgical Site Infection. London: RCOG Press; 2008 Oct. Disponible en: lurlhttps://www.ncbi.nlm.nih.gov/pubmed/21698848

11. Galli R. et al. Laparoscopic approach in perforated appendicitis: increased incidence of surgical site infection? Surg Endosc. 2013 Aug;27(8):2928-33. Disponible en: https://www.ncbi.nlm.nih.gov/pubmed/23443482

2. Takada T, et al. The Role of Digital Rectal Examination for Diagnosis of Acute Appendicitis: A Sys- 
tematic Review and Meta-Analysis. Marson L, editor. PLOS ONE. 2015 Sep 2;10(9):e0136996. Disponible en:http://journals.plos.org/plosone/article?id=10. 1371/journal.pone.0136996

13. Wu W-T, Tai F-C, Wang P-C, Tsai M-L. Surgical Site Infection and Timing of Prophylactic Antibiotics for Appendectomy. Surg Infect (Larchmt). 2014 Dec;15(6):781-5.

14. Instituto Nacional de Estadísticas y Censos. Anuario de Estadísticas de Salud: Camas y Egresos Hospitalarios 27 2018. Disponible en: https://www.ecuadorencifras.gob.ec/ camas-y-egresos-hospitalarios/

15. Rael Ruiz S, López Pérez MV. Factores de riesgo que 28 contribuyen a la infección del sitio quirúrgico. Metas Enferm jul/ago 2016; 19(6): 14-20.

16. Giesen LJX, van den Boom AL, van Rossem CC, den Hoed PT, Wijnhoven BPL. Retrospective Multicenter 29. Study on Risk Factors for Surgical Site Infections after Appendectomy for Acute Appendicitis. Dig Surg. 2017;34(2):103-7.

17. Kasatpibal N, Nørgaard M, Sørensen HT, Schønheyder HC, Jamulitrat S, Chongsuvivatwong V. Risk of surgical site infection and efficacy of antibiotic prophylaxis: A cohort study of appendectomy patients in Thailand. BMC Infect Dis. 2006 Jul 12;6.

18. Brill A, Ghosh K, Gunnarsson C, Rizzo J, Fullum T, Maxey C, et al. The effects of laparoscopic cholecystectomy, hysterectomy, and appendectomy on nosocomial infection risks. Surg Endosc Other Interv Tech. 2008 Apr;22(4):1112-8.

19. Billoro BB, Nunemo MH, Gelan SE. Evaluation of antimicrobial prophylaxis use and rate of surgical site infection in surgical ward of Wachemo University Nigist Eleni Mohammed Memorial Hospital, Southern Ethiopia: Prospective cohort study. BMC Infect Dis. 2019 Apr 2;19(1).

20. Winfield RD, Reese S, Bochicchio K, Mazuski JE, Bochicchio G V. Obesity and the risk for surgical site infection in abdominal surgery. In: American Surgeon. Southeastern Surgical Congress; 2016. p. 331-6.

21. Noorit P, Siribumrungwong B, Thakkinstian A. Clinical prediction score for superficial surgical site infection after appendectomy in adults with complicated appendicitis. World J Emerg Surg. 2018 Jun 18;13(1).

22. Kotaluoto S, Pauniaho SL, Helminen M, Kuokkanen H, Rantanen T. Wound healing after open appendectomies in adult patients: A prospective, randomised trial comparing two methods of wound closure. World J Surg. 2012 Oct;36(10):2305-10.

23. Xiao Y, Shi G, Zhang J, Cao J-G, Liu L-J, Chen T-H, et al. Surgical site infection after laparoscopic and open appendectomy: a multicenter large consecutive cohort study. Surg Endosc. 2015 Jun 11;29(6):1384-93.

24. Jaschinski T, Mosch CG, Eikermann M, Neugebauer EA, Sauerland S. Laparoscopic versus open surgery for suspected appendicitis. Cochrane Database Syst Rev. 2018 Nov 28;(11).

25. Cedillo Villamagua AG. Profilaxis de infección del sitio quirúrgico en apendicectomía mediante infiltración local aleatoria de metronidazol, hospital Vicente Corral Moscoso. Cuenca, 2012. Universidad de Cuenca; 2014.

26. Guanchún Guanchún MA. Validación de la escala de Alvarado y criterios del síndrome de respuesta inflamatoria sistémica para apendicitis aguda complicada, hospital Vicente Corral Moscoso, Cuenca 2014. Universidad de Cuenca; 2019.

. Sohn M, Hoffmann M, Hochrein A, Buhr HJ, Lehmann KS. Laparoscopic Appendectomy Is Safe. Surg Laparosc Endosc Percutan Tech. 2015 Jun;25(3):e90-4.

. Cho M, Kang J, Kim I, Lee KY, Sohn S-K. Underweight Body Mass Index as a Predictive Factor for Surgical Site Infections after Laparoscopic Appendectomy. Yonsei Med J. 2014 Nov 1;55(6):1611.

. Fukuda H. Patient-related risk factors for surgical site infection following eight types of gastrointestinal surgery. J Hosp Infect. 2016 Aug;93(4):347-54.

Recibido: 8 de octubre de 2020

Aceptado: 22 de octubre de 2020 